\section{Can Parents Treat their Anxious Child using CBT? A Brief Report of a Self-Help Program}

\section{Abstract}

Objective: We developed and tested a self-help program with minimal therapist involvement for parents of anxious children.

Method: The program focused on transfer of control from therapist to parents of children with moderate anxiety, and consisted of two therapist-led workshops, a Facebook group, and Cool Kids manuals for parents and children. The sample consisted of 20 families, and 17 completed treatment.

Results: After treatment, intent-to-treat analyses indicated that $65 \%$ of the children were free of all anxiety disorders. The corresponding figure for completers was $76.5 \%$.

Conclusion: Our results suggest that parent-based self-help groups focusing on transfer of control may be a cost-effective way of providing treatment to children with moderate anxiety.

Keywords: Child anxiety; Parent-based self-help; Cognitive behavior therapy; Transfer of control; Cool kids program

Received: January 14, 2016; Accepted: February 10, 2016; Published: February 19, 2016

\section{Practitioner Points}

- Parent-based self-help groups may be promising for moderate levels of child anxiety.

- We tested a group program based on two parent workshops, and the Cool Kids manuals.

- Twenty families with moderately anxious children aged 7-13 years were enrolled.

- ITT revealed that $65 \%$ of children were free of all anxiety diagnoses post treatment.

- Results should be interpreted with caution due to the limited sample size.

Anxiety disorders have an early age of onset and are highly prevalent. As many as $23 \%$ of all youth have fulfilled diagnostic criteria for at least one anxiety disorder when they reach young adulthood [1, 2]. Most treatment studies of child anxiety apply cognitive behavioral therapy (CBT; for a meta-analysis [3]). Evidence suggests that therapist-led CBT programs have been effective, e.g. a recent meta-analysis reported that $59.4 \%$ of children were free of all anxiety disorders following treatment [4]. However, existing programs are labor intensive, thus making the dissemination of these treatments to everyday practices in

\section{Barbara Hoff Esbjørn, Bianca Munkebo Christiansen, Monika Walczak, Sofie Wille Østergaard and Sonja Breinholst}

\author{
Department of Psychology, University of \\ Copenhagen, Denmark
}

Corresponding author: Barbara Hoff Esbjørn

\section{barbara.hoff@psy.ku.dk}

Department of Psychology, University of Copenhagen, $\emptyset$ ster Farimagsgade 2A, 1353 København K, Denmark.

Tel: $+45-35324905$

Citation: Esbjørn BH, Christiansen BM, Walczak M, et al. Can Parents Treat their Anxious Child using CBT? A Brief Report of a Self-Help Program. Acta Psychopathol. 2016, 2:1.

the community difficult. Self-help programs have been developed as part of a stepped care approach, and the general effects are promising [5]. However, only few studies have investigated if anxious children may be successfully treated by their parents in a self-help setting. One study [6] examined the effect of treatment delivered by parents to their anxious child using three different formats. All groups received parental based self-help via the book Help Your Anxious Child and the Cool Kids manual for the children. They only differed in the degree of therapeutic contact; one group received one weekly telephone call by the therapist, a second group received one weekly email, and in the third group parents themselves had to take the initiative to contact the therapist if and when needed. The authors found that $80 \%$ of the children in the first group were free of all anxiety disorders post treatment, while the effect was $33 \%$ and $30 \%$ for the second and third group, respectively. This finding suggests that a treatment with some possibility of talking to a therapist can be applied with self-help. Another study, applied an internet-based format with minimal therapist support [7], for treating children with anxiety. The families received ten weekly internet-based child sessions and six parent sessions. They were 
offered weekly online contact with a therapist and a telephone contact (15-30 minutes) on two occasions. When comparing the results from the treatment condition with waitlist controls, the study found a small but significantly higher reduction in anxiety symptoms in treatment condition. At post-treatment $30 \%$ of the children receiving treatment were free of their primary anxiety, and $16.7 \%$ were free of all anxiety disorders, however, these percentages had increased to $75 \%$ and $60.7 \%$ respectively at 6 months follow-up. Investigations have also been conducted with respect to how treatment effect may be increased. A recent meta-analysis [3] suggested that treatment with an active focus on transfer of control from therapists to parents, where parents learn how to encourage brave behavior and exposure with their anxious child, was significantly more effective at 1 year follow-up than treatments without this focus. In Denmark, we have a long tradition for involving parents in their child's everyday life, also in treatment of mental health problems. Building on this tradition, we set out to develop and pilot-test, a parent-based self-help group with minimal therapist involvement for treating childhood anxiety.

The aim of the study was to develop and test a treatment which could be applied in a stepped care approach in a community setting. The treatment therefore targeted children whom the clinician considered to be moderately disabled by their anxiety disorder. Based on previous studies, we hypothesized that the parent-based self-help groups would have a moderate but positive effect.

\section{Method}

\section{Participants and procedures}

Parents self-referred their child for treatment in a university setting. Families participated in pre- and post-treatment assessment. Inclusion criteria were 1) age of child: 7-13 years, 2 ) the child had a primary diagnosis of generalized or separation anxiety disorder, specific or social phobia, 3) the primary anxiety disorder was moderate (Clinical severity rating (CSR): 4-7) rated by parent, child and clinician, 4) the child had no intellectual disabilities, 5) families had not received prior treatment for their child's anxiety disorder, and 6) one parent was a native speaker of Danish.

Assessors were blinded to diagnostic status, and progression of treatment. The study was approved by the Institutional Review Board at the Department of Psychology, University of Copenhagen, and complies with ethical standards for children enrolled in psychological research. Written informed consent was obtained from all families. At intake 20 families were enrolled, 1 declined participation, and 2 dropped out, thus, 17 families completed treatment.

\section{Treatment}

The treatment format was self-help groups for parents of 5-8 anxious children. It consisted of two therapist-led workshops ( 2 hours each), the Cool Kids manuals for parent and child [1], and a Facebook group. The level of training of the therapists varied; however, all therapists had worked at least one year with CBT programs for anxious children prior to being responsible for carrying out the workshops. Also, they were supervised by a specialist in CBT for anxious children throughout the project. The first workshop was conducted prior to commencing treatment. Therapists provided psycho-education regarding anxiety, the role of parents in maintenance of symptoms, and an introduction to the Cool Kids manuals in the workshops. Main goals were to: transfer control to parents, and enhance their self-efficacy beliefs regarding their ability to achieve success. This was done by encouraging discussions about everyday challenges and successes with their child and reinforcing when parents had spontaneously successfully applied treatment techniques. In the interval between the first and second workshop, the families followed the Cool Kids manual, working through one session a week. The second workshop was conducted after five weeks. During this workshop therapists inquired about challenges and helped identify solutions to problems as well as help the parents set up and adjust the child's exposures. Finally, the remaining sessions were discussed. Each week, the parents received a standardized Facebook notice regarding the session of the week. Parents were also encouraged to discuss the sessions with each other when needed via Facebook. If needed the therapist would guide the parents to the appropriate section in the manual regarding the mentioned problem. Parents conducted the treatment over a 12 week period.

\section{Measures}

Anxiety Disorders Interview Schedule for DSM-IV, Child and Parent Versions (ADIS-IV-C/P) [8] measures anxiety disorders in children. It consists of two independent interviews administered to child and parents, separately. Symptoms are rated on a CSR scale from 0-8. A score of $\geq 4$ indicates clinical anxiety. We applied a composite diagnosis based on child and parent information. Interviews were administered by trained staff who received supervision throughout the project. Throughout the project a supervisor who was trained in psychiatric assessment viewed the videotapes of the interviews and approved the given diagnoses or lack thereof.

Revised Children's Anxiety and Depression Scale (RCADS) [9] was used to assess anxiety symptoms. RCADS consists of 47-items loading on five anxiety and one depression subscale. We applied child, mother and father ratings. Symptoms were rated on a 4-point scale. Higher scores indicate higher severity. The current study applied the total anxiety score based on the sum of the anxiety subscales.

Depression, Anxiety and Stress Scale (DASS 21) [10] was used to assess parental levels of psychopathology. DASS 21 is a 21 item self-report questionnaire designed to measure the severity of symptoms common to depression, anxiety and stress in adults. We report on the depression and anxiety subscale for mothers and fathers independently.

\section{Results}

Twenty families participated in the study, 17 completed treatment within the 12 week period. The children's mean age was $10.4 \pm 1.96$ years (range: $7-13$ years). Forty percent were female. All participants were middle class Caucasians. Eighteen 
children were Danish, one child was half Danish and half Polish, and one child was half Danish and half Israeli. Primary diagnoses were: generalized anxiety disorder (50\%), specific phobia (25\%), separation anxiety disorder (20\%), and social phobia (5\%). Average primary diagnosis CSR was $5.8 \pm 0.8$. The majority of the children $(60 \%)$ had comorbidity, with $8(40 \%)$ fulfilling criteria for two diagnoses, 3 (15\%) children for three, and 1 (5\%) child fulfilling criteria for four diagnoses. Most of the comorbid diagnoses were other anxiety disorders, while two of the children had comorbid ADHD diagnoses, and three children had comorbid oppositional defiant disorder.

Twenty mothers and seventeen fathers were assessed at intake. Mothers' and fathers' mean age was $39.9 \pm 4.2$ and $44.0 \pm 6.8$ years, respectively. Eighty percent were married. More than half $(65 \%)$ of the mothers and $58 \%$ of the fathers had completed a college education or higher. The annual income of $70 \%$ of the mothers and $76 \%$ of the fathers exceeded 500.000 DKK (approx. 75.000 US dollars). Seven mothers and four fathers reported having had psychological difficulties in the past. Parental reports of their current symptom levels of anxiety and depression, as measured using DASS 21 , indicated that the vast majority were within the normal range. Out of 20 mothers, only one reported mild levels of anxiety, one mild levels of depression and another mother reported moderate levels of depressive symptoms. Out of 17 fathers, only one showed mild levels of anxiety and depression.

\section{Treatment effect}

Treatment outcome on anxiety diagnoses was analysed using intent-to-treat (ITT), and completer analysis. In ITT analyses the missing scores at post-treatment were replaced with the corresponding value from intake. A positive outcome "free of all anxiety" was defined as not fulfilling criteria for any anxiety disorder at post-treatment. ITT analyses revealed that 13 children (65\%) were free of all anxiety. The corresponding percentage using completer analyses was $76.5 \%$. A series of paired-samples t-tests were conducted to compare the levels of anxiety symptomatology in the participants before and after treatment. ITT analyses showed that there was a significant decline in mean scores of participants' anxiety levels before $(M=36.50, S D=14.92)$ and after $(M=21.00, S D=13.44)$ treatment; $\mathrm{t}(19)=5.06, p=0.000$, based on child reports. A significant difference in the scores at pre- and post-treatment was also found in parent reported child symptomatology, with $(M=35.15, S D=12.99)$ before and $(\mathrm{M}=19.65, \mathrm{SD}=14.01)$ after treatment; $\mathrm{t}(19)=5.06, p=0.000$, for mother-reported symptoms, and $(M=30.24, S D=10.84)$ before and $(M=21.94, S D=10.16)$ after treatment; $t(19)=3.28, p=0.005$, for father-reported symptoms. Means and standard deviations for self and parent reported anxiety symptoms and $p$-values for both ITT and completers are shown in Table 1.

\section{Discussion}

The study set out to explore the treatment effect of parentbased self-help groups with minimal therapist involvement on moderate levels of childhood anxiety disorders. Our hypothesis was, that treatment would be effective, albeit only moderately
Table 1 Change in self and parent reported anxiety symptomatology from pre- to post-treatment.

\begin{tabular}{|c|c|c|c|}
\hline $\begin{array}{c}\text { Anxiety symptoms } \\
n\end{array}$ & \multicolumn{3}{|c|}{ Pre-treatment Post-treatment } \\
\hline $\begin{array}{c}\text { Child } \\
\text { ITT 20 } \\
\text { Completer } 17\end{array}$ & 36.5014 .92 & 21.0013 .44 & $\mathrm{P}<0.001$ \\
\hline $\begin{array}{c}\text { Mother } \\
\text { ITT 20 }\end{array}$ & 36.1816 .07 & 17.9411 .95 & $\mathrm{P}<0.001$ \\
\hline $\begin{array}{c}\text { Completer } 17 \\
\text { Father }\end{array}$ & 33.7112 .87 & 19.6514 .01 & $\mathrm{P}<0.001$ \\
\hline $\begin{array}{c}\text { ITT 17 } \\
\text { Completer 13 }\end{array}$ & 30.2410 .84 & 21.9410 .16 & $\mathrm{P}<0.01$ \\
\hline
\end{tabular}

Note: Anxiety symptoms based on total RCADS scores; $p$-values based on Paired-sample t-tests

as suggested by previous studies with less than weekly therapist contact $[6,7]$. ITT analyses revealed that $65 \%$ of the children were free of all anxiety following a 12 week treatment period. Externalising disorders did not have any influence on response to treatment for anxiety disorders in our study. Another Danish study found $48.2 \%$ of the children to be free of all anxiety following therapist-led Cool Kids treatment [11]. A meta-analysis reported $59.4 \%$ of children to be free of all anxiety following CBT [4]. In comparison, our results were unexpectedly positive. We offer two explanations. First, our children had moderate levels of anxiety. Children suffering from severe anxiety were excluded. Previous studies included these, which may explain our better results. However, we intended to test a program that could be applied as a first step in a stepped-care approach, which was costeffective and easily applied in everyday settings. Stepped care programs have gained increased attention and recognition within clinical psychology in general. The programs are typically defined by a low intensity phase, with self-help elements, and a high intensity phase for those who do not respond sufficiently, often being therapist-assisted [5]. A low intensity phase, as our selfhelp program, has the advantage of being easily applied in the community, as it enables problems to be detected and treated at an early stage, thereby preventing serious clinical levels. This may potentially reduce the overall societal costs for treating severe psychopathology [12].

The second explanation for our high success rate could be that parents self-referred their child for treatment. We therefore expect a bias towards higher motivation in parents for participating in treatment. Additionally, the majority of the parents in our study were well educated and had moderate to high incomes. Our sample cannot be considered representative of the general population. Nonetheless, this type of treatment may be well suited for parents who themselves seek assistance. However, it should be noted that early therapist-conducted school-based interventions may also be applied to socioeconomically disadvantaged families with positive effects [13]. Before drawing any conclusions regarding the effect of parentbased self-help groups in disadvantaged families, further studies need to be conducted. One could expect that more therapist 
activity, e.g. small videos of how techniques should be applied, posted on Facebook, might be needed if this program was to be applied to disadvantaged families.

\section{Limitations and Future Directions}

Although our study has potential strengths regarding the applicability due to the limited number of resources required to provide treatment to moderately anxious children, the paper is not without limitations. One such limitation is the small sample size, which may prevent us from drawing firm conclusions regarding the findings before the results have been replicated in a larger sample. Another limitation is that the current study does not address the issue of acceptability by parents and children. We have not assessed if parents found, e.g. the Face-book group useful or not, also we do not know if parents felt adequately instructed to perform treatment by attending the workshops. Finally, we cannot know how parents felt about acting as therapists for their child, nor do we know how children felt about having their parents act as their therapists. In future studies of the self-help groups, these issues should be addressed for instance via interviews of the participating parents and their children. Another future direction concerns which studies are needed before conclusions regarding the applicability of the parent self-help groups can be drawn. We suggest two routes of investigation. One such route would contain a feasibility study conducted in a community setting for which the program has been developed. As treatment in the current study took place at a specialized anxiety clinic in a university setting, findings cannot automatically be generalized to community settings. Future research should test the applicability of the program in these settings. Another route would be to conduct a treatment trial comparing different treatment formats. We hypothesize that adding therapist-led workshops increase the self-efficacy beliefs in the parents. Therefore this should be assessed in a study comparing, e.g. waitlist control, pure bibliotherapy and our selfhelp groups including therapist-led workshops, bibliotherapy and Facebook group.

\section{Conclusion}

We tested a parent-based self-help group program for parents of children with moderate levels of anxiety disorders. Our results are corresponding with treatment effects found in therapist led CBT programs. Albeit, the sample is biased towards higher motivation, education and less anxiety than other samples, our results suggest that this type of treatment may be effective for children with moderate levels of anxiety whose parents seek treatment for their child. 


\section{References}

1 Rapee RM, Wignall A (2002) Cool Kids Program: Family Version session guide. Sydney, Australia: Macquarie University Anxiety Research Unit.

2 Copeland WE, Angold A, Shanahan L, Costello EJ (2014) Longitudinal Patterns of Anxiety From Childhood to Adulthood: The Great Smoky Mountains Study. Journal of the American Academy of Child \& Adolescent Psychiatry 53: 21-33.

3 Manassis K, Lee TC, Bennett K, Zhao XY, Mendlowitz S, et al. (2014) Types of parental involvement in CBT with anxious youth: A preliminary meta-analysis. Journal of consulting and clinical psychology 82: 1163.

4 James AC, James G, Cowdrey FA, Soler A, Choke A (2013) Cognitive behavioural therapy for anxiety disorders in children and adolescents (Review). The Cochrane Collaboration.

5 Williams C, Martinez R (2008) Increasing access to CBT: stepped care and $C B T$ self-help models in practice. Behavioural and Cognitive Psychotherapy 36: 675-683.

6 Lyneham HJ, Rapee RM (2006) Evaluation of therapist-supported parentimplemented CBT for anxiety disorders in rural children. Behaviour Research and Therapy 44: 1287-1300.

7 March S, Spence SH, Donovan CL (2009) The efficacy of an internet- based cognitive-behavioral therapy intervention for child anxiety disorders. Journal of Pediatric Psychology 34: 474-487.

8 Silverman WK, Albano AM (1996) Anxiety Disorders Interview Schedule for DSM-IV: Parent interview schedule (Vol. 1). Oxford University Press.

9 Chorpita BF, Yim L, Moffitt C, Umemoto LA, Francis SE (2000) Assessment of symptoms of DSM-IV anxiety and depression in children: a revised child anxiety and depression scale. Behaviour Research and Therapy 38: 835-855.

10 Lovibond PF, Lovibond SH (1995) The structure of negative emotional states: Comparison of the Depression Anxiety Stress Scales (DASS) with the Beck Depression and Anxiety Inventories. Behaviour Research and Therapy 33: 335-343.

11 Arendt K, Thastum M, Hougaard E (2015) Efficacy of a Danish version of the Cool Kids program: a randomized wait-list controlled trial. Acta Psychiatrica Scandinavica 1-13.

12 Bodden DH, Dirksen CD, Bogels SM (2008) Societal burden of clinically anxious youth referred for treatment: a cost-of-illness study. Journal of abnormal child psychology 36: 487-497.

13 Mifsud C, Rapee RM (2005) Early Intervention for Childhood Anxiety in a School Settting: Outcomes for an Economically Disadvantaged Population. Journal of the American Academy of Child \& Adolescent Psychiatry 44: 996-1004. 